\title{
Evaluation of thin film adhesion to a compliant substrate by the analysis of progressive buckling in the fragmentation test
}

\author{
J. Andersons ${ }^{\mathrm{a}, *}$, S. Tarasovs ${ }^{\text {a }}$, Y. Leterrier ${ }^{\mathrm{b}}$ \\ a Institute of Polymer Mechanics, University of Latvia, Rīga LV 1006, Latvia \\ ${ }^{\mathrm{b}}$ Laboratoire de Technologie des Composites et Polymères (LTC), Ecole Polytechnique Fédérale de Lausanne (EPFL), CH 1015 Lausanne, Switzerland
}

\section{A R T I C L E I N F O}

Article history:

Received 6 January 2008

Received in revised form 27 October 2008

Accepted 28 October 2008

Available online 6 November 2008

\section{Keywords:}

Adhesion

Coatings

Interfaces

Silicon oxide

\begin{abstract}
A B S T R A C T
The interface toughness of a thin coating/compliant substrate system is estimated based on the evolution of coating buckle patterns in the fragmentation test. The linear density of coating buckles as a function of applied strain is determined experimentally for a $\mathrm{SiO}_{\mathrm{x}}$ coating deposited on a polyethylene terephthalate film. A three-dimensional non-linear finite element model is developed to simulate the process of buckle formation in a single narrow coating strip. The elastic energy released during buckling-driven delamination is obtained from the energy balance in the system before and after the buckling event. Both the interface adhesion and the total energy release rate, which includes the plastic dissipation in the substrate during debonding, are evaluated. The apparent interfacial toughness, equal to $15 \mathrm{~J} / \mathrm{m}^{2}$ at the onset of buckling, is found to increase with strain. This is tentatively explained by the probabilistic features of the buckle accumulation process, reflected also in the random locations of buckles evolving towards a log-normal distribution of buckle spacings at high strains.
\end{abstract}

(c) 2008 Elsevier B.V. All rights reserved.

\section{Introduction}

Interlaminar adhesion is a characteristic of primary importance for ensuring mechanical integrity of laminated structures. A number of experimental methods to estimate the interfacial toughness of thin film/ substrate systems have been elaborated, see reviews [1-3], bucklingdriven delamination being one of them. In flexible, organic electronics components, a large mismatch of elastic moduli between stiff films and compliant substrates is common. The effect of the mismatch on the shape of buckling-driven delamination has been determined [4,5] and the relations for steady state energy release rate (ERR) obtained [6,7]. In order to initiate bucking with subsequent delamination, a sufficient level of compressive stress in the film has to be reached. This can be accomplished by mechanical (e.g. bending the coated substrate $[5,8]$ ) or physical (temperature variation [5], hydrogen treatment [9]) means.

A similar loading mechanism leading to buckle formation is seen in the fragmentation test [3]. The test is usually applied to evaluate the interfacial shear strength between the coating and the substrate based on the crack density at saturation. The latter is reached at a relatively high tensile strain. However, during tensile loading of the coated film, compressive transverse stresses arise in the coating fragments due to Poisson's ratio mismatch between the substrate and the coating. The compressive stresses cause progressive buckling and delamination of coating fragments transverse to the tensile loading direction [10-14].

\footnotetext{
* Corresponding author.

E-mail address: janis.andersons@pmi.lv (J. Andersons).
}

The observation of the debonding process can be used to determine the interfacial adhesion characteristics. The interfacial fracture energy is evaluated in [11] as the energy released by the coating per unit interface area, based on the average dimensions of the buckled zone and the buckling onset stress. Only the buckled part of the coating is considered in the energy balance, an approach pertinent to stiff substrates [1,7]. It is assumed in [12] that buckling commences with debonding at the free edges of coating strips governed by mode II fracture toughness. The latter is evaluated by an elastic interfacial stress analysis at the experimentally determined buckling onset stress.

Buckling-driven delamination tunnels rapidly through a fragment if the adhesion is low to moderate [11-13]. Hence, the critical ERR can be estimated from the energy balance in the system before and after the buckling event [15], using the finite fracture mechanics (FFM) approach. FFM implies that if a crack of a well-defined geometry appears spontaneously at a given load level (i.e. its propagation is fast and the finer details of crack development are not important), then the actual crack growth process, normally the subject of fracture mechanics, can be neglected and the crack appearance treated as a fracture event. Such a fracture event occurs when the finite amount of energy released per unit crack area is equal to (or exceeds) the critical ERR. The interpretation of toughness as derived by FFM arguments is discussed in [16-18]. It is claimed that the FFM approach is best applicable to similar fracture events, e.g. progressive cracking.

We apply the FFM analysis to progressive buckling of a $103 \mathrm{~nm}$ thick $\mathrm{SiO}_{\mathrm{x}}$ coating on a polyethylene terephthalate (PET) film studied in [10]. The buckling process is shown to evolve gradually over a strain 
a

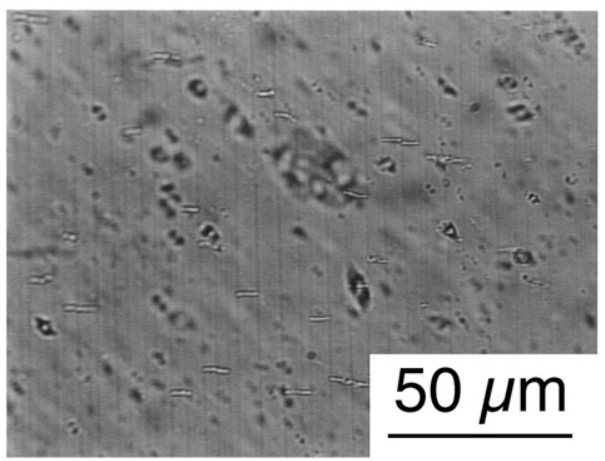

b

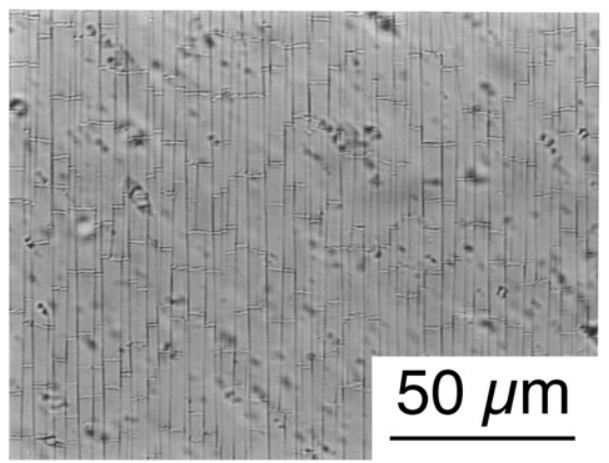

Fig. 1. Cracked morphology of the $\mathrm{SiO}_{\mathrm{x}}$ coating on the PET substrate under 7\% (a) and $15 \%$ (b) uniaxial tensile strain (the loading direction is parallel to the fiducial mark on the micrographs). Compressive buckles are visible as horizontal features delimited by vertical tensile cracks.

range that apparently depends on interface imperfections, scatter in toughness, and buckle interaction. Probabilistic features of the buckle pattern evolution are discussed.

\section{Materials and methods}

The material investigated is a $12 \mu \mathrm{m}$ thick extruded and biaxially stretched PET film, coated by means of reactive evaporation with a

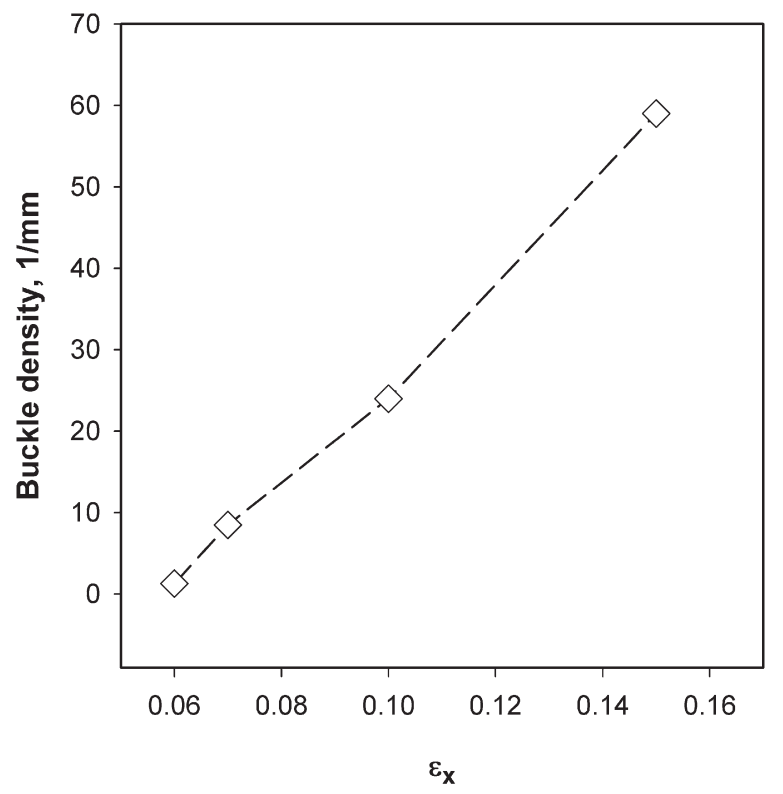

Fig. 2. Buckle density in $\mathrm{SiO}_{\mathrm{x}}$ coating fragments on PET as a function of applied tensile strain.

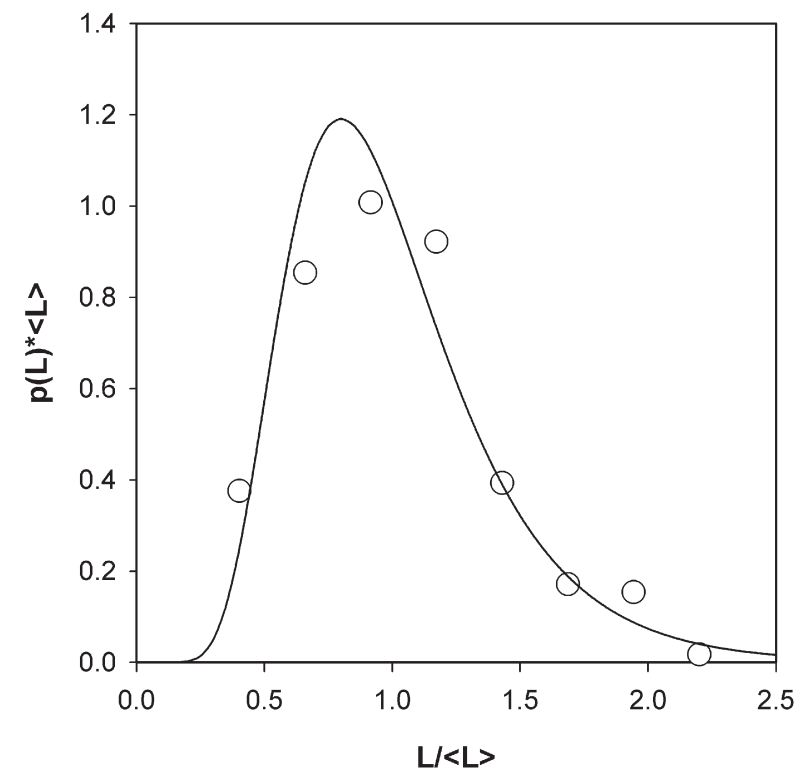

Fig. 3. Normalized buckle spacing distribution density in $\mathrm{SiO}_{\mathrm{x}}$ coating fragments at $15 \%$ tensile strain. The line represents an approximation of the data by the log-normal distribution.

$103 \mathrm{~nm}$ thick $\mathrm{SiO}_{\mathrm{x}}$ layer, where $\mathrm{x}=1.66 \pm 0.03$ as measured from X-ray photoelectron spectroscopy.

Fragmentation tests [3] under uniaxial loading were performed at room temperature on $40 \times 10 \mathrm{~mm}^{2}$ rectangular samples using a miniature tensile tester with displacement control (Minimat, Rheometric Systems) in situ in an optical microscope (Olympus SH-2). A small prestrain was applied to the film samples to assure proper alignment that induced an uncertainty of about $0.2 \%$ nominal strain in the recorded strain values. The crack development during straining at a constant strain rate of $2.110^{-4} \mathrm{~s}^{-1}$ was video recorded via a coupledcharge device camera connected to the microscope. Later, the video was played back and the crack pattern was analysed at selected strains. In the present study, attention was paid to the occurrence of buckling features within individual coating fragments as shown in Fig. 1. The linear buckling density (i.e. the number of buckles per unit fragment length) was determined as the inverse of the average spacing between adjacent buckles.

The linear buckle density as a function of the applied strain is presented in Fig. 2. The buckle spacing distribution in $\mathrm{SiO}_{\mathrm{x}}$ coating fragments at a tensile strain of 0.15 is shown in Fig. 3 in normalized coordinates.

\section{Model of buckling-driven delamination in a coating strip}

\subsection{Elementary estimate of interfacial toughness}

Consider a coating fragment of width $w$ adhering to the substrate subjected to tensile strain, Fig. 4 . The transverse compressive stress in

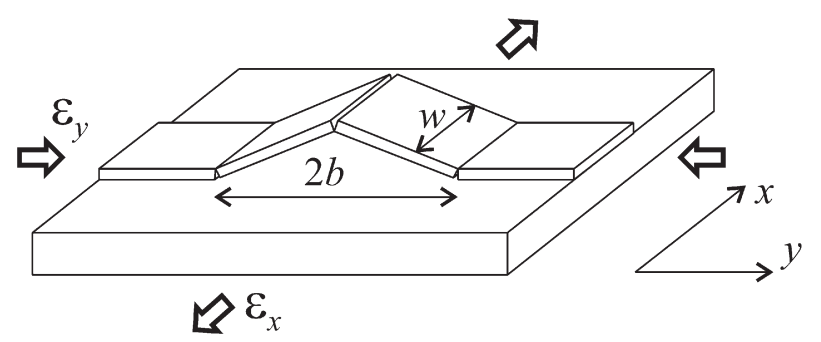

Fig. 4. Coating fragment with a delaminated and cracked buckle. 
the coating fragment is related to the longitudinal tensile strain, $\varepsilon_{x}$, applied to the substrate and to the mechanical strain $\varepsilon_{x}^{c}$ acting in the coating. The compressive transverse stress in the coating arises due to the difference in transverse contraction between the substrate and the coating, resulting from the mismatch of Poisson's ratios and tensile strains, and is given to a first approximation by:

$\sigma_{y}^{c}=\sigma_{r}^{c}+E_{c}\left(-\nu_{s} \varepsilon_{x}+\nu_{c} \varepsilon_{x}^{c}\right)$

where $\sigma_{r}^{c}$ is the residual stress in the coating, $E$ stands for Young's modulus, $\nu$ is Poisson's ratio, and subscripts $s$ and $c$ relate to substrate and coating properties, respectively.

Buckling onset is observed at a relatively advanced fragmentation stage when the length of the tensile stress recovery zone, $\lambda$, exceeds the fragment width, $\lambda>w$. As a result, the applied strain is not fully transferred to the coating fragment, $\varepsilon_{x}^{c}<\varepsilon_{x}$, and the strain distribution can be approximated by [13]

$\varepsilon_{x}^{c}=\frac{\varepsilon_{x}}{2 \lambda^{2}} x(w-x)$

with the origin of the $x$ axis taken at the fragment edge. As $\varepsilon_{x}^{c}=\varepsilon_{x}^{c}(x)$ varies along the fragment width, so does the compressive stress $\sigma_{y}^{c}$ reaching the maximum (compressive) value at fragment edges where $\varepsilon_{x}^{c}=0$. Therefore, a buckle is more likely to originate at the fragment edge. The average value of the compressive coating stress over the fragment width is evaluated by Eqs. (1) and (2) as

$\bar{\sigma}_{y}^{c}=\sigma_{r}^{c}+E_{c} \varepsilon_{x}\left(-\nu_{s}+\frac{\nu_{c}}{12}\left(\frac{w}{\lambda}\right)^{2}\right)$.

A buckling event releases the strain energy from the buckled coating zone of extent $2 b$ and also from the stress perturbation zones adjacent to the buckle. For a compliant substrate, the perturbation zone of the coating stress extends over a distance $l_{p}$ from buckle fronts [7]

$l_{p}=\left(1+\frac{\bar{E}_{c}}{\bar{E}_{s}}\right) h_{c}$

where the overbar denotes the plane strain modulus and $h_{c}$ is the coating thickness. Assuming for simplicity that the compressive stress is fully released due to the buckling event from a coating zone of linear extent $2 b+2 l_{p}$ we estimate the ERR associated to delamination as

$G_{a d h}=\frac{\left(\bar{\sigma}_{y}^{c}\right)^{2} h_{c}}{2 E_{c}}\left(1+\frac{l_{p}}{b}\right)$.

\subsection{Numerical analysis}

A three-dimensional non-linear finite element (FE) model is used to analyze the process of buckling driven delamination in a coating strip. The substrate is modeled as an elastic-plastic material with isotropic strain hardening, while a linear elastic behavior of the coating is assumed. As cohesive cracks usually develop in coating fragments upon buckling (shown schematically in Fig. 4), we simplify the modeling task by assuming complete detachment of the debonded part of the coating. This is accomplished by removing the parts of the coating corresponding to buckles from the FE model at the appropriate analysis steps, by means of reducing the coating material modulus to zero.

The numerical analysis is performed in two steps. In the first step, residual stresses are generated by a temperature change and the prescribed tensile strain is applied by enforcing suitable displacement boundary conditions. In the second step, part of the coating corresponding to the experimentally determined buckle width is removed as described above thus modelling the buckling event.
The elastic energy released during debonding is evaluated through the energy balance of the system before and after the buckling event. The elastic energy, $U_{i}$, due to residual stresses and the external work, $W$, applied to the system during the first step of analysis are equal to the sum of stored elastic energy $U_{e l}$ and dissipated energy $D_{p l}$ at the end of the step

$U_{e l}+D_{p l}=W+U_{i}$

No external work is done during the second step of analysis. Part of the elastic energy released during the process of coating delamination is dissipated in plastic deformation of the substrate, and the remaining part is attributed to the new surface creation

$\Delta U_{e l}+\Delta D_{p l}+G_{a d h} \cdot \Delta A=0$

where $\Delta U_{e l}, \Delta D_{p l}$ denote the increments of elastic energy and dissipation during the second step, $\Delta A$ is the debonded area, and $G_{a d h}$ is the ERR associated to debonding, i.e. the interfacial toughness. The latter is evaluated using Eq. (7) as

$G_{a d h}=-\frac{\Delta U_{e l}+\Delta D_{p l}}{\Delta A}$.

The total energy release rate, that includes both the energy spent on the new surface creation and the plastic dissipation in the substrate accompanying debonding, is given by

$G_{c}=-\frac{\Delta U_{e l}}{\Delta A}$.

Appropriate symmetry conditions are enforced to reduce the $\mathrm{FE}$ model size. For the initial stages of buckling, when the average buckle spacing exceeds the perturbation zone of the coating stress $l_{p}$ (evaluated by FEM), the lateral size of the model is chosen slightly larger than $l_{p}$. However, when the average distance between buckles at a load level of interest becomes comparable to or smaller than $l_{p}$, buckle interaction is accounted for by adjusting the size of the FE model and introducing the pre-existing debonded buckle at a specified location in the first step of the numerical analysis. Fig. 5 shows a part of the FE model upon delamination and detachment of the buckled coating.

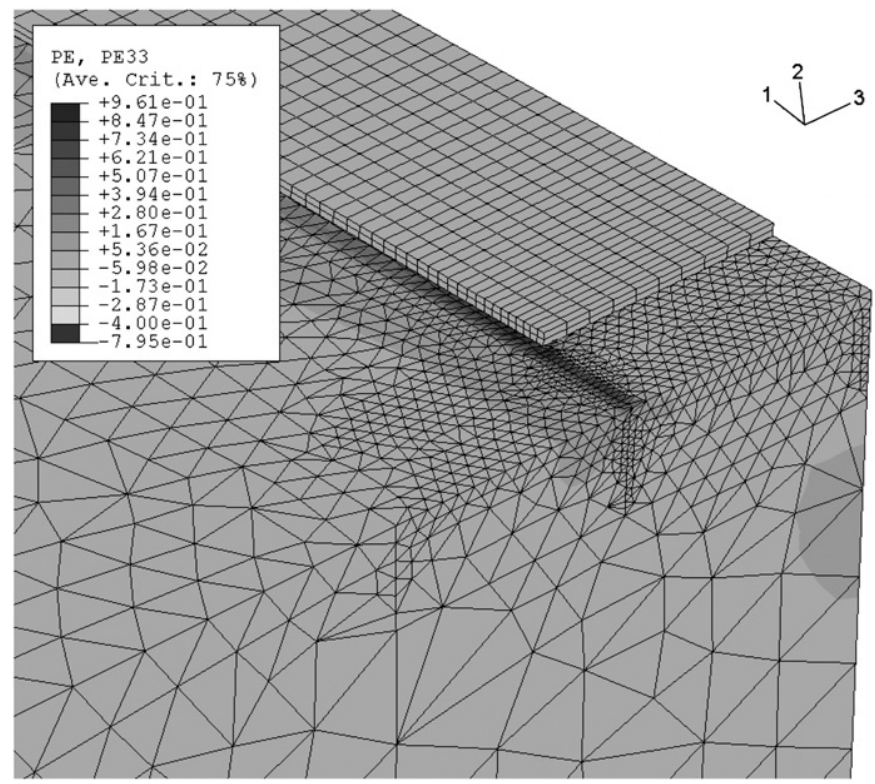

Fig. 5. Finite element model showing the plastic deformation upon delamination and detachment of the buckled coating. 


\section{Results and discussion}

The finite element code ABAQUS was used for numerical analysis. The FE model comprised about 30000 elements. The coating was modelled using quadratic brick elements and quadratic tetrahedral elements were used for the substrate. The coating was attached to the substrate by a surface-to-surface "tie" constraint, available in ABAQUS. The thickness of the substrate in the FE model was taken 120 times larger than the coating thickness according to the $\mathrm{SiO}_{\mathrm{x}} / \mathrm{PET}$ film geometry. The bottom plane of the substrate was fixed in the vertical (thickness-wise) direction in order to prevent spurious bending under the applied load due to limited FE model dimensions.

The FE analysis of the $\mathrm{SiO}_{\mathrm{x}}$ coating with a single buckle together with a linear interpolation of the experimental buckle density vs strain data of Fig. 2 revealed that the onset of buckle interaction is reached at ca $12 \%$ tensile strain. The length of the stress relaxation zone is about $12.5 \mu \mathrm{m}$, virtually independent of the applied strain as suggested by the stress recovery data of Fig. 6 , and the respective critical buckle density amounts to $40 \mathrm{~mm}^{-1}$.

ERR analysis is performed for the four applied strain levels at which buckle density is measured, and the results are presented in Fig. 7. The residual stress in the coating is estimated at -272 MPa [13]. The first buckles appeared at about $6 \%$ strain, which corresponds to an interfacial adhesion of $G_{a d h} \sim 15 \mathrm{~J} / \mathrm{m}^{2}$ as evaluated by Eq. (8). Upon load increase, buckles develop at other sites with higher apparent adhesion. The data trend in Fig. 7 suggests that a saturation level is approached at larger strains with an interface adhesion of about $54 \mathrm{~J} /$ $\mathrm{m}^{2}$. The full energy release rate, Eq. (9), which includes plastic dissipation in the substrate near the delamination zone, amounts to $G_{c}$ $\sim 60 \mathrm{~J} / \mathrm{m}^{2}$ at the applied strain of $6 \%$ and increases up to $160 \mathrm{~J} / \mathrm{m}^{2}$ at $15 \%$ strain.

In order to apply the analytical model of Section 3.1, we rely on the value of $\lambda$ obtained for the considered material system in [13] and amounting to $\lambda \approx 40 h_{c}$. Upon numerical evaluation of the terms in Eq. (3), it ensues that the correction due to the tensile strain in the coating fragment is small, hence $\bar{\sigma}_{y}^{c} \approx \sigma_{c}^{r}-v_{s} E_{c} \varepsilon_{x}$. It is interesting to note that the elementary estimate for $G_{a d h}$ obtained using Eq. (5) follows rather closely the FE results as seen in Fig. 7. However, the linear elastic analysis leading to Eq. (4) considerably underestimates the stress relaxation length, producing $l_{p}=1.9 \mu \mathrm{m}$.

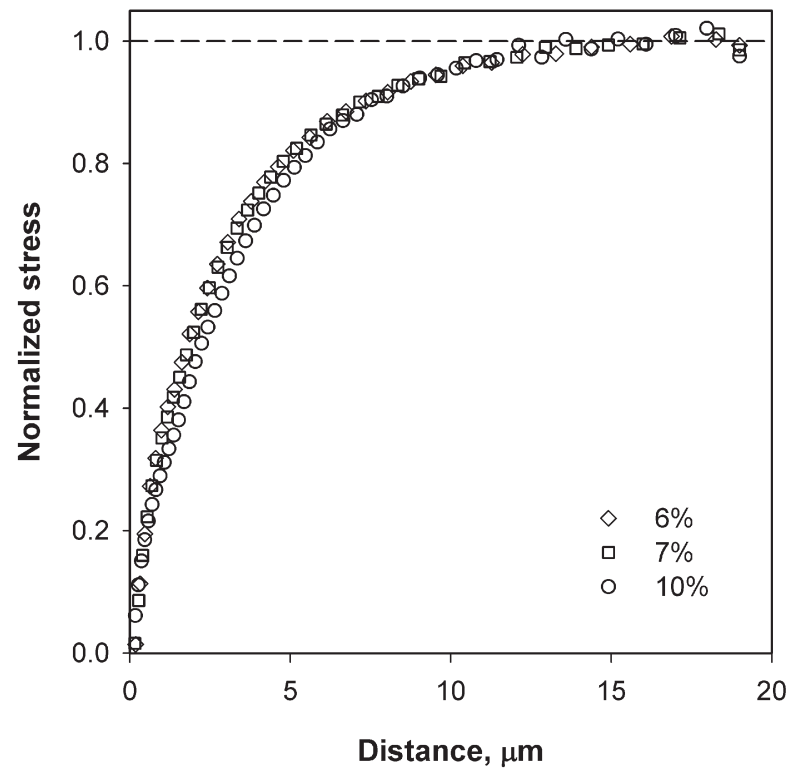

Fig. 6. Evolution of the average compressive coating stress $\bar{\sigma}_{y}^{c}$, normalised by its far-field value, along the coating fragment obtained by FE analysis under different applied strains. The distance is measured from the buckle front.

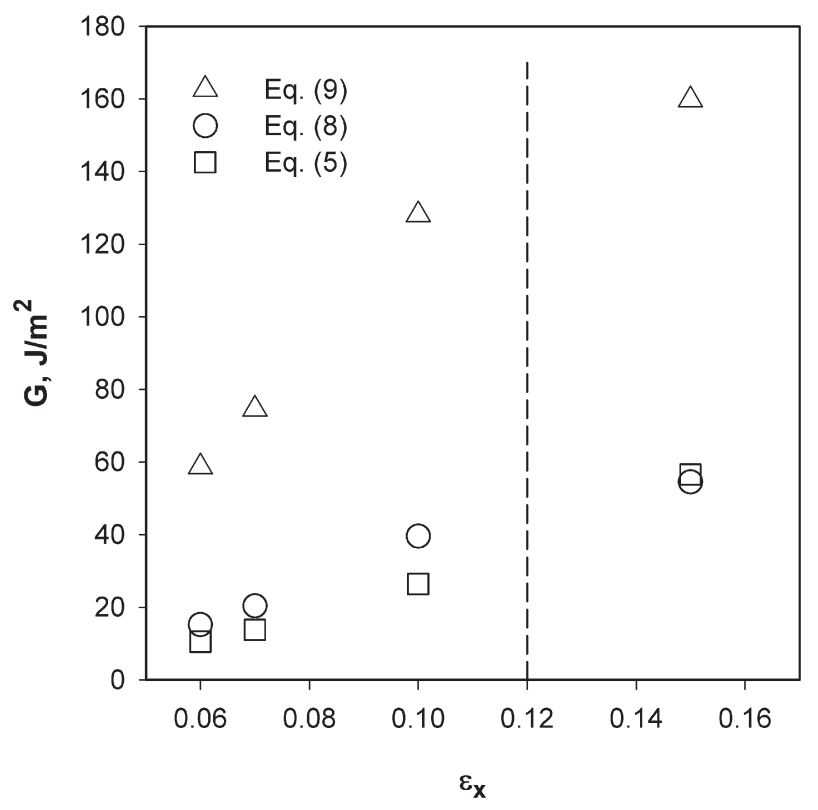

Fig. 7. FFM estimate of the energy release rate by buckling driven delamination as a function of the applied tensile strain in $\mathrm{SiO}_{\mathrm{x}} / \mathrm{PET}$ fragmentation test. The interfacial adhesion $G_{a d h}$ evaluated by Eq. (8) is shown by (O), $G_{a d h}$ according to Eq. (5) by ( $\square$ ), full energy release rate $G_{c}$, Eq. (9), by $(\Delta)$. The dashed line indicates the onset strain for buckle interaction.

The apparent dependence of $G_{a d h}$ on strain presumably reflects the heterogeneity of interface properties. The random locations of buckles, see [10] and Fig. 1, as well as the scatter in buckle spacing represented in Fig. 3 indicate that a probabilistic mechanism influences the progressive buckling process. It follows from theoretical arguments of e.g. [19-22] that progressive random fragmentation evolves towards a log-normal fragment size distribution under rather general conditions. In the case of constrained cracking of composite materials in tension, such a distribution has been verified for binary fragmentation at large strains [23]. Note that the buckle spacing distribution of interacting buckles, Fig. 3, also is close to log-normal. By contrast, the deterministic coating wrinkling process (retaining bonding to the substrate) due to loss of stability generates uniformly spaced buckle patterns [24,25] (with the buckling wavelength dependent on fragment width [26]).

Coating fragments are likely to buckle at imperfection sites, which could be low interface adhesion, geometrical imperfections of coating or substrate [27] etc, when the ERR made available by the formation of a buckle exceeds the local interfacial fracture toughness. We have neglected the variation of the ERR along the coating fragment (associated to a local variability in the geometrical parameters and deformability of the coating, substrate, and interphase) by considering only a perfectly planar fragment geometry and uniform mechanical properties. Therefore, all the material and geometrical variability effects related to the interface are contained in the apparent interfacial adhesion. The probabilistic features of progressive buckling are likely to be governed by Poisson-Weibull statistics that is applied to stochastic fracture modelling of brittle solids, e.g. fibres and fibrous composites [28], in tension. Probabilistic modelling of the buckle accumulation process is the subject of further work. However, for a stochastic buckle accumulation process, the critical ERR value at buckling onset is likely to provide a conservative estimate of the adhesion, as it effectively probes the toughness at the weakest spot of the interface.

The accuracy of the $G_{a d h}$ estimates obtained using the proposed model is affected by the simplifying assumptions introduced above by the model geometry. The presence of open buckles in the $\mathrm{SiO}_{\mathrm{x}} / \mathrm{PET}$ system at large strains has been verified in [29]. We presumed that the 
buckling process produces predominantly open buckles at all strains, and neglected the residual strain energy present in the partially detached coating fragments. Another simplification relates to replacing, in the numerical analysis, the coating segmented by cohesive cracks by a single coating strip. Thus the constraining effect of adjacent fragments on the stress relief caused by buckling-driven delamination is neglected in the model. This leads to an increase of calculated $G_{a d h}$. However, the error in $G_{a d h}$ estimate at buckling onset is less than $10 \%$, as suggested by additional FEM calculations accounting for the proximity of neighbouring fragments to the debonding one. Lastly, we assumed that the delamination runs strictly along the coating/substrate interface producing smooth debonded surfaces. Should the actual delamination process produce microdamage, such as crazing or microcracking, of the substrate, the dissipated energy would also be included in the effective $G_{a d h}$.

\section{Conclusions}

A finite fracture mechanics based model is developed to evaluate the adhesion of a thin brittle coating to a compliant substrate using buckling patterns observed during a fragmentation test. The model is applied to $\mathrm{SiO}_{\mathrm{x}} / \mathrm{PET}$ test data and enables interfacial toughness estimates to be obtained. In the initial stage of the progressive buckling characterized by widely spaced, non-interacting buckles, the apparent toughness is found to markedly increase with applied strain. This is tentatively explained by the probabilistic nature of buckling events, related to the local variability in geometrical, deformability, and adhesion parameters of the system. An estimate of the adhesion toughness $G_{a d h}$ equal to $15 \mathrm{~J} / \mathrm{m}^{2}$ is obtained at buckling onset.

\section{Acknowledgements}

Part of this work has been supported by the EC-funded project FlexiDis (contract IST-2004-4354). The authors would also like to thank Lawson Mardon Packaging for providing film samples.

\section{References}

[1] J.W. Hutchinson, Z. Suo, Adv. Appl. Mech. 29 (1992) 63.

[2] A.A. Volinsky, N.R. Moody, W.W. Gerberich, Acta Mater. 50 (2002) 441.

[3] Y. Leterrier, Prog. Mater. Sci. 48 (2003) 1.

[4] G. Parry, J. Colin, C. Coupeau, F. Foucher, A. Cimetière, J. Grilhé, Acta Mater. 53 (2005) 441.

[5] A.A. Abdallah, D. Kozodaev, P.C.P. Bouten, J.M.J. den Toonder, U.S. Schubert, G. de With, Thin Solid Films 503 (2006) 167.

[6] B. Cotterell, Z. Chen, Int. J. Fract. 104 (2000) 169.

[7] H.-H. Yu, J.W. Hutchinson, Int. J. Fract. 113 (2002) 39

[8] Z. Chen, B. Cotterell, W. Wang, E. Guenther, S.-J. Chua, Thin Solid Films 394 (2001) 202.

[9] A. Pundt, E. Nikitin, P. Pekarski, R. Kirchheim, Acta Mater. 52 (2004) 1579.

[10] Y. Leterrier, L. Boogh, J. Andersons, J.-A.E. Månson, J. Polym. Sci. Part B: Polym. Phys. 35 (1997) 1449.

[11] H. Bordet, M. Ignat, M. Dupeux, Thin Solid Films 315 (1998) 207.

[12] B.E. Alaca, M.T.A. Saif, H. Sehitoglu, Acta Mater. 50 (2002) 1197.

[13] J. Andersons, Y. Leterrier, G. Tornare, P. Dumont, J.-A.E. Månson, Mech. Mater. 39 (2007) 834.

[14] S. Iwamoto, S. Ochiai, H. Okuda, T. Inoue, ISIJ Int. 47 (2007) 930.

[15] J. Andersons, S. Tarasovs, Y. Leterrier, Key Eng. Mater. 348-349 (2007) 329

[16] Z. Hashin, J. Mech. Phys. Solids 44 (1996) 1129.

[17] J.A. Nairn, Fifth International Conference on Deformation and Fracture of Composites, London, 1999, p. 1.

[18] J.A. Nairn, in: A. Kelly, C. Zweben (Eds.), Comprehensive Composite Materials, vol. 2, Pergamon, 2000, p. 403.

[19] A.N. Kolmogorov, Doklady Akad. Nauk. SSSR, 31, 1941, p. 99, [in Russian].

[20] B. Epstein, J. Franklin Inst. 244 (1947) 471.

[21] G. Ouillon, D. Sornette, A. Genter, C. Castaing, J. Phys. I 6 (1996) 1127.

[22] J.A. Åström, Adv. Phys. 55 (2006) 247.

[23] J. Andersons, Y. Leterrier, Prob. Eng. Mech. 22 (2007) 285.

[24] A.L. Volynskii, S. Bazhenov, O.V. Lebedeva, N.F. Bakeev, J. Mater. Sci. 35 (2000) 547.

[25] Z.Y. Huang, W. Hong, Z. Suo, J. Mech. Phys. Solids 53 (2005) 2101.

[26] S. Tarasovs, J. Andersons, Int. J. Solids Struct. 45 (2008) 593.

[27] J.W. Hutchinson, M.Y. He, A.G. Evans, J. Mech. Phys. Solids 48 (2000) 709.

[28] S.L. Phoenix, I.J. Beyerlein, in: A. Kelly, C. Zweben (Eds.), Comprehensive Composite Materials, vol. 1, Pergamon, 2000, p. 559.

[29] Y. Leterrier, P. Sutter, J.-A.E. Månson, J. Adhes. 69 (13) (1999). 